\title{
Définition et contrôle du lait stérilisé
}

\author{
par \\ Jean PIEN
}

PREMIÈRE PARTIE

\section{DEFINITION DU LAIT STERILISE}

Il n'existe, à l'heure actuelle, aucune définition internationale du lait stérilisé. C'est là une lacune grave, car cette absence de définition est à l'origine de la confusion qui règne dans le langage courant, comme dans certains textes réglementaires et même dans la littérature scientifique, quant au sens à donner à ces mots.

Dans quelques pays, en revanche, il existe une définition légale du lait stérilisé ; mais ces différentes définitions locales sont le plus souvent divergentes, ce qui signifie que la plupart d'entre elles ne sont pas satisfaisantes.

Il paraît donc indispensable de tenter de dégager les principes d'une définition du lait stérilisé, puis d'en déduire les termes d'un texte général (qui pourrait, d'ailleurs, servir de base à une réglementation internationale).

Tel est l'objet de la présente étude.

$$
*
$$

\section{PRINCIPES D'UNE DEFINITION CORRECTE}

Dans le cas qui nous occupe, définir c'est prescrire les caractères d'un objet en fonction $d u$ but poursuivi. C'est exprimer ce qu'il doit être pour être conforme à sa destination, à sa raison d'être. Appliquons cette notion au problème du lait stérilisé.

Pourquoi prépare-t-on du lait stérilisé ? A quel souci cette fabrication correspond-elle ?

Il est facile de répondre : le lait stérilisé doit se conserver. Il s'agit de prolonger considérablement la possibilité de le mettre en vente et de l'utiliser. 
Donc, le lait stérilisé doit se maintenir, le plus longtemps possible, sans altération, c'est-à-dire en conservant ses qualités initiales.

Mais une telle définition ne serait pas suffisante, car elle ne donne qu'une confirmation du but, sans indiquer le moyen de l'atteindre.

Il est donc nécessaire d'aller plus loin.

\section{Premier principe : la stérilité du lait stérilisé est inutile}

Les altérations que nous voulons éviter sont dues, essentiellement, à l'activité des micro-organismes. Il faut donc, théoriquement, les détruire (ce qui signifie que les laits stérilisés devraient être stériles).

Or, la meilleure connaissance que nous avons depuis vingt ans de la physiologie microbienne, nous permet d'affirmer que la présence de micro-organismes vivants n'implique pas toujours, ni nécessairement, l'apparition des altérations. Pour qu'il y ait altération, en effet, il est nécessaire que les individus microbiens soient présents en grand nombre. Un seul germe vivant dans un grand volume de liquide et ne s'y développant pas, est incapable, par son métabolisme individuel, d'entraîner une altération décelable ou mesurable. Pour atteindre cet " état de grand nombre " indispensable, il faut que les micro-organismes puissent proliférer, c'est-à-dire se reproduire. Or, dans quelques milieux naturels, comme le lait, certains germes sont dans l'impossibilité de proliférer soit que certaines formes végétatives ne puissent se reproduire, soit que des formes particulières (certaines spores) soient incapables de germer. Cette impossibilité de proliférer peut tenir à des facteurs intrinsèques intéressant la cellule microbienne ou à des facteurs extrinsèques résultant de la composition chimique ou biochimique du milieu ou encore d'influences physiques antérieures.

Il résulte de ces connaissances relativement nouvelles cette conséquence très importante que, dans certaines circonstances, la présence de micro-organismes vivants, peu nombreux et non proliférants, est parfaitement compatible avec l'aptitude à une bonne conservation (1).

Donc : la conservabilité n'implique pas la stérilité.

D'autres auteurs ont depuis longtemps exprimé la même opinion (notamment Tentoni [20]).

(1) Il est même parfaitement établi que la multiplication des germes ayant survécu à la stérilisation n'entraîne pas toujours une modification physicochimique du lait se traduisant par une altération (Hermier [12]).

Cependant nous ne considérerons pas comme acceptable, dans le lait stérilisé, la présence d'une flore résultant de la prolifération de germes quelconques, même si elle n'entraîne pas d'altération. 
Une conséquence de ce premier principe en vue de la définition du lait stérilisé sera, non pas que le lait stérilisé doit être stérile mais qu'il ne doit pas renfermer de micro-organismes susceptibles d'y proliférer.

\section{Deuxième principe : la stérilité absolue est pratiquement impossible à atteindre}

Une objection vient immédiatement à l'esprit : en admettant, ce qui est exact, que la présence de certains germes vivants, peu nombreux et non proliférants, soit compatible avec une parfaite conservabilité, pourquoi ne pas tendre à la stérilité totale et pourquoi ne pas l'exiger, ce qui résoudrait tous les problèmes ?

La réponse est simple : la stérilité totale, dont on vient de démontrer qu'elle est inutile, est impossible à atteindre pratiquement.

En effet :

Il est bien connu que, dans une culture pure d'une espèce sporulée déterminée, il est possible de rencontrer des spores de résistances thermiques différentes. Cette probabilité s'accroît évidemment lorsqu'il s'agit de mélanges d'espèces différentes comme il s'en présente dans les contaminations spontanées d'un milieu liquide tel que le lait cru. Les cellules les plus résistantes sont les moins nombreuses dans les conditions moyennes et il est évident que la probabilité de rencontrer des germes d'une thermorésistance exceptionnelle croît avec l'importance quantitative de la contamination.

Pour obtenir la «stérilité », il faudrait évidemment soumettre le produit à des conditions thermiques capables de détruire les organismes les plus résistants. Or, quelles que soient les précautions prises, il est inévitable que le produit à stériliser renferme plus ou moins de spores très thermorésistantes. Donc, les conditions thermiques à mettre en œuvre risquent toujours de devoir être très sévères.

Il est difficile de déterminer quelles doivent être ces conditions, car elles dépendent de la nature et du nombre de spores, de leur degré de thermorésistance et, en technologie pratique, des appareils utilisés (efficacité bactéricide ou « effet stérilisant »).

Tous les chiffres rencontrés dans la littérature sont, pour ces raisons, difficiles à interpréter. Ce que l'on peut dire, c'est qu'ils sont toujours très sévères (en température ou en durée) quand ils résultent d'études pratiques ayant pour objet d'atteindre la stérilité totale, avec une marge de sécurité suffisante.

Ainsi, dans l'industrie des antibiotiques où le problème se pose de stériliser absolument de grands volumes de milieux, Rambaud [19], utilisant un matériel perfectionné de stérilisation continue, adopte $7 \mathrm{mn}$ à $140^{\circ} \mathrm{C}$ pour des milieux limpides et acides. Cet auteur cite 
les chiffres donnés antérieurement par Pfeifer et Vojnovich [17] et qui s'étendent de $5 \mathrm{mn}$ à $135^{\circ} \mathrm{C}$ dans le cas des milieux limpides jusqu'à $13 m n$ à $163^{\circ} \mathrm{C}$ dans le cas des milieux troubles.

Il est donc certain que si l'on avait à stériliser de grands volumes de lait ne devant plus renfermer une seule spore vivante, il faudrait, pour ce milieu trouble et voisin de la neutralité, des conditions thermiques de cet ordre ou plus sévères encore.

Or, il est parfaitement connu qu'en opérant ainsi on altérerait très fortement les caractères organoleptiques du lait et même sa stabilité. Dans de nombreux cas, le lait (même parfaitement neutre) serait coagulé et dans tous les cas il serait fortement coloré, bruni et présenterait une saveur désagréable de brûlé. De toute façon, il serait inutilisable.

Voilà pourquoi, pratiquement, il est impossible d'envisager de préparer du lait qui soit absolument stérile dans tous les cas. Il ne s'agit pas, en l'occurence, d'une impossibilité de détruire la totalité des germes vivants (on y parvient bien dans l'industrie des antibiotiques) mais du fait qu'il serait inacceptable, dans le cas du lait, d'appliquer les conditions techniques qui le permettraient.

C'est pourquoi nous disons que la stérilité absolue de volumes quelconques de lait est impossible à atteindre (sans risquer de détruire le lait lui-même).

Remarque: Une nouvelle objection se présente immédiatement à l'esprit :

S'il est vraiment impossible pratiquement d'atteindre la stérilité du lait, comment se fait-il cependant que l'on rencontre tous les jours dans le commerce, des laits stérilisés d'excellente qualité et de longue conservation ? Apparemment, de tels laits sont stériles. Comment concilier cette observation avec ce qui vient d'être dit ?

Il convient de faire deux réponses à cette observation pertinente :

\section{a) INFLUENCE DU DIVISIONNEMENT SUR LA PROBABILITÉ DE STÉRILITÉ}

Lorsque, dans l'industrie des antibiotiques, on cherche à atteindre la stérilité absolue d'une cuve contenant $50 \mathrm{~m}^{3}$ de milieux de culture, il faut appliquer à ce volume entier les conditions thermiques sévères qui correspondent à la destruction des quelques spores les plus thermorésistantes de toutes (ou même de l'unique spore plus résistante que toutes les autres). Si cet objectif n'était pas atteint, la réussite de la fabrication serait compromise.

Dans le cas du lait, les conditions sont différentes : on n'aura jamais à "stériliser " le contenu d'une cuve de $50 \mathrm{~m}^{3}$. Ce volume sera divisionné par exemple en 50000 récipients de 1 1. Or si, dans le volume total, il n'existe que quelques spores très thermorésistantes, elles ne seront évidemment pas présentes dans chacun des 50000 récipients mais seulement dans quelques-uns (voire même dans un seul). 
Dans ces conditions, en appliquant aux 50000 récipients contenant le lait des conditions thermiques moins sévères que celles qui correspondraient à la stérilité absolue, c'est-à-dire, par exemple, compatibles avec le maintien des propriétés organoleptiques du produit, on aboutit au double résultat suivant :

1) Certains récipients ne sont pas stériles. Ce sont ceux qui contiennent une ou plusieurs spores très thermorésistantes, non détruites par les conditions thermiques moins sévères qui ont été adoptées.

2) D'autres récipients (la grande majorité) ne renfermant pas ces spores très thermorésistantes (ou renfermant seulement des espèces moins résistantes) sont stériles et il est théoriquement possible d'affirmer que leur stérilité est absolue.

Tout le problème pratique du lait stérilisé revient donc à déterminer quel est le pourcentage relatif de ces deux classes de récipients. Or, ce pourcentage relatif dépend de deux facteurs :

1) La probabilité de la présence des spores très thermorésistantes. Cette probabilité est liée au degré de contamination initiale du produit. Plus le produit a été contaminé, plus le risque de rencontrer des spores très résistantes est grand. Et, moins ces spores exceptionnelles sont nombreuses, moins il y aura de récipients non stériles.

2) Le degré de sévérité des conditions de chauffage, compatibles avec la conservation des caractères organoleptiques du produit. Plus ces conditions pourront être sévères, plus la stérilisation sera efficace et plus le nombre de récipients stériles sera grand.

On voit donc que la «stérilité » du lait divisionné en récipients, est le résultat d'une probabilité statistique concernant l'importance du pourcentage du nombre de récipients vraiment stériles.

On rencontrera donc, dans le commerce, une très grande majorité de récipients réellement stériles et quelques autres qui ne le sont pas.

Comment se fait-il, cependant, que l'on ne trouve pratiquement jamais chez les détaillants de lait "non stérile », altéré, etc. (exception faite du cas accidentel de récipients non étanches ayant été secondairement contaminés) ? La deuxième "réponse » que nous présentons maintenant va nous éclairer sur ce point.

\section{b) NON DÉVELOPPEMENT DES SPORES SURVIVANTES}

Nous avons montré plus haut que la conservabilité n'impliquait pas la stérilité parce que, dans le lait stérilisé, peuvent survivre des spores hautement thermorésistantes, incapables de se développer dans le lait, donc de l'altérer. Ainsi, même après de longues périodes de stockage et même après incubation, ces laits, renfermant encore des spores ayant survécu à la stérilisation, ne sont pas modifiés et restent d'excellente qualité. C'est pourquoi, dans le commerce, (et 
même, il faut bien le dire, à l'occasion du contrôle courant) ces laits passent inaperçus puisqu'ils ne se distinguent pas de ceux qui sont absolument stériles.

Une réserve, cependant, reste à faire :

On peut admettre que les récipients non absolument stériles à l'issue du traitement thermique relativement modéré qu'ils ont subi, se divisent en deux classes:

- Ceux qui ne renferment que des spores non susceptibles de se développer dans le lait (ce sont ceux dont nous venons de parler et dont la présence passe inaperçue, même dans le cas du contrôle courant). Ils sont assimilables aux récipients contenant du lait absolument stérile.

- Ceux qui renferment des spores très thermorésistantes, certes, et capables de germer et de proliférer dans le lait, donc, éventuellement, de l'altérer. La pratique montre que ces récipients sont très rares et que, dans un lot donné, leur pourcentage est infime ou nul. Cette circonstance ne constitue aucune entrave pratique au développement de l'industrialisation et de la vente du lait stérilisé, pas plus que les rares accidents qui peuvent résulter de la défaillance ou du défaut d'étanchéité d'un emballage.

Voilà donc pourquoi, en dépit du fait que la stérilité absolue d'un volume quelconque de lait est une impossibilité pratique, on rencontre dans le commerce une très grande majorité de récipients de lait stérilisé de bonne qualité, de bonne conservation c'est-à-dire conformes à ce que l'on attend d'eux.

Après avoir montré que la conservabilité, caractère essentiel du lait stérilisé, n'impliquait pas la stérilité (ou, ce qui revient au même, que la stérilité était inutile) puis, que cette stérilité était pratiquement irréalisable en valeur absolue sans risquer de détruire le lait lui-même, il nous reste à dire que la stérilité, quand elle est possible, est indémontrable donc incontrôlable.

\section{Troisième principe : la stérilité n'est pas contrôlable}

Le contrôle de la stérilité revient à rechercher la présence de micro-organismes vivants et à aboutir à une réponse négative.

Or, de quels moyens pratiques dispose-t-on pour cette recherche ? Uniquement ceux qui permettent la mise en évidence des manifestations de la vie c'est-à-dire, soit la reproduction, se traduisant par une prolifération, soit la détection des produits du métabolisme, soit les deux.

Dans le cas d'un très petit nombre de cellules vivant dans un grand volume de liquide, sans proliférer, ces détections correspondent à une impossibilité pratique. L'addition de "suppléments 》 peut, dans certains cas, permettre à des germes vivants de proliférer 
dans le lait et de l'altérer, (Hermier [12], Kaplan [14]). Mais les germes qui seront insensibles à l'apport de ces suppléments (ou, ce qui revient au même, à la transplantation dans un milieu nutritif plus favorable) passeront inaperçus et seront considérés comme morts ou absents. On peut donc dire dans ce cas qu'un échantillon renfermant des germes vivants serait déclaré "stérile " puisqu'il serait impossible de mettre ces germes en évidence.

En fait, on ne pourra jamais trancher entre les deux termes suivants : ou bien l'échantillon est stérile, ou bien il a été impossible de déceler la présence de certaines cellules vivantes, si les techniques actuelles ne le permettent pas.

Au reste, en admettant même qu'une technique améliorée donne, demain, le moyen de dépister à coup sûr la présence éventuelle de tout micro-organisme vivant, la réponse ne serait valable que pour la prise d'essai soumise à l'examen et non pour le volume entier du récipient. Et si la technique permettait l'examen du contenu du récipient entier, la réponse ne serait pas valable pour les autres récipients du lot entier.

Donc, à l'impossibilité technique de décider de la stérilité d'une prise d'essai ou d'un récipient, s'ajoute l'impossibilité statistique de conclure à la stérilité d'un lot entier. Dans les deux cas, une réponse négative ne correspond qu'à une certaine probabilité de stérilité.

En conclusion : si l'on définissait le lait stérilisé comme devant être stérile, cette définition serait incontrôlable, donc sans utilité puisqu'il ne serait pas possible d'affirmer qu'elle a été respectée (non pas pour la raison que la stérilité ne serait pas possible théoriquement, mais parce qu'il serait impossible de la prouver pratiquement).

\section{$\stackrel{*}{*}$ \\ ELABORATION DE LA DEFINITION DU LAIT STERILISE}

Nous avons donc jusqu'ici dégagé un certain nombre de principes qui vont nous guider maintenant dans l'élaboration de la définition du lait stérilisé.

Nous savons en effet :

- que le lait stérilisé, conformément à son but, doit se conserver le plus longtemps possible (pour faciliter sa distribution, sa mise en vente, etc.) ;

- que, pour atteindre ce résultat, il ne doit pas renfermer de germes vivants susceptibles de l'altérer c'est-à-dire de micro-organismes capables de proliférer dans le lait stérilisé ;

- que, en conséquence, il n'est pas nécessaire que le lait stérilisé soit stérile (cette stérilité inutile étant, par ailleurs, impossible 
à réaliser pratiquement dans le cas du lait et, d'autre part, incontrôlable).

De sorte que la véritable définition du lait stérilisé devrait au moins reposer sur le fait de l'absence totale d'altération au cours de sa conservation, impliquant l'absence de proliférations microbiennes c'est-à-dire finalement, l'absence de germes capables d'y proliférer (ce qui n'exclut pas nécessairement la présence de quelques germes vivants, incapables de se développer dans le lait stérilisé, voulant exprimer par là, comme nous l'avons montré, que la stérilité n'est pas nécessaire, donc pas exigible). nition.

Voilà donc un point de départ très clair pour une bonne défi-

Cependant, il faut bien reconnaître que si ce point de départ est nécessaire, il n'est pas entièrement suffisant. En effet :

\section{1) Limitation du nombre acceptable de germes vivants}

Nous admettons donc la présence, dans le lait stérilisé, de spores ayant résisté au chauffage et incapables de germer, donc de proliférer dans le lait.

Peut-on accepter qu'il y en ait un nombre quelconque, par exemple très élevé, si leur présence s'avère sans inconvénient ? En fait, il s'agit là d'un faux problème puisque l'expérience a montré depuis longtemps que les spores très thermorésistantes sont fort peu nombreuses dans le lait cru (même dans les pays chauds) : de l'ordre de une par ml au maximum (1).

Divers auteurs (Burton, Buttiaux, Galesloot, etc.) estiment que, dans le lait correctement stérilisé, il subsiste moins de 50 ou moins de 100 spores vivantes par litre de lait.

Nous n'avons donc pas à redouter, dans le lait stérilisé (non recontaminé) la présence d'un nombre élevé de spores vivantes et l'on serait tenté, théoriquement, de ne pas s'en préoccuper et de passer sous silence, dans la définition, une limitation quelconque de ce nombre. Nous persistons cependant à penser qu'il convient d'envisager cette limitation.

En effet :

Nous ne devons pas oublier que parmi les germes ayant survécu à la stérilisation (notamment à une stérilisation trop peu sévère) il peut exister, à côté des spores incapables de germer, des germes parfaitement capables de se développer mais dont la présence ne se

(1) On admet d'ailleurs que le nombre total de spores (y compris toutes celles qui sont faciles à détruire dans des conditions de stérilisation n'altérant pas les caractères organoleptiques du lait) ne dépasse pas quelques centaines par ml. 
traduit pas toujours par une modification physico-chimique du lait (Hermier [12]).

Dans une définition uniquement basée sur l'absence d'altération, c'est-à-dire sur la stabilité physico-chimique du lait stérilisé au cours d'incubations appropriées, de tels germes pourraient donc proliférer sans faire rejeter l'échantillon.

Cette perspective n'est pas acceptable, d'abord parce qu'il n'est pas absolument démontré que ce développement n'entraînerait pas à la longue, dans certains cas, des modifications imprévisibles (donc une altération), ensuite parce que la nature de ces germes proliférants et altérants n'est pas connue à priori et peut être suspecte ; enfin parce que si nous acceptons que quelques spores vivantes puissent survivre dans le lait stérilisé, ce ne peut être qu'à la faveur d'une thermorésistance sévère de quelques individus isolés, incapables ultérieurement de se développer, et non pas comme le résultat d'une prolifération de survivants susceptibles de se multiplier rapidement.

Donc, toute question d'hygiène mise à part (voir ci-après) si nous avons le droit d'accepter la présence de spores survivantes, non capables de proliférer dans le lait au cours d'une incubation par exemple, nous avons aussi le devoir d'en limiter le nombre, et cela sera d'autant plus facile que, dans la pratique courante, ce nombre est déjà, précisément et naturellement, très limité (de l'ordre de 1 spore par $\mathrm{ml}$ ou même beaucoup moins) (Franklin et al. [6]).

Les bactériologistes français qui se sont penchés sur ce problème, ont estimé qu'une limite supérieure fixée à 5 spores revivifiables par $\mathrm{ml}$ de lait stérilisé, était raisonnable et devait très largement couvrir tous les cas normaux (1).

Remarque : Les partisans de la "stérilité " du lait stérile voudraient, bien entendu, que cette « norme » soit ramenée à zéro, autrement dit, qu'il n'y ait aucune spore vivante revivifiable sur milieux artificiels dans $1 \mathrm{ml}$ de lait. Or, l'expérience que nous avons de ce genre de contrôles montre qu'il en est très souvent ainsi, même dans le cas de laits non stériles et sans que, pour autant, l'on puisse affirmer la stérilité. En effet : si le lait ne renferme qu'une seule de ces spores survivantes dans $10 \mathrm{ml}$ de lait, l'examen de $1 \mathrm{ml}$ n'aura qu'une chance sur dix de donner un résultat positif. Un tel lait non stérile, mais parfaitement satisfaisant, sera donc, le plus souvent, déclaré stérile, à moins de procéder à des contrôles portant sur des dizaines de repiquages de $1 \mathrm{ml}$. D'autre part, si le principe de la stérilité obligatoire était admis, le lait que nous venons de

(1) Ces spores qui n'auront pas germé dans le lait stérilisé, même au cours des incubations prévues à l'occasion du contrôle, seront mises en évidence sur des milieux nutritifs appropriés où elles donneront des colonies dénombrables (voir plus loin). 
citer en exemple (et qui, encore une fois, est parfaitement satisfaisant) aurait une chance sur dix d'être refusé.

Puisque, comme nous l'avons longuement exposé plus haut, la notion de stérilité du lait stérilisé doit être rejetée, la raison commande d'accepter la présence de quelques spores survivantes et d'imposer une limite raisonnable quant à leur nombre.

Enfin, pour en terminer avec cette notion, et pour répondre à une ultime objection, il faut dire qu'il n'y a aucun risque que ces quelques spores soient le résultat de la prolifération, au cours des incubations, de germes ayant résisté à la stérilisation. Si tel était le cas, on sait très bien que le nombre constaté lors du contrôle serait considérable (même éventuellement sans altération du lait) et que cette circonstance serait très facile à mettre en évidence par un simple examen microscopique direct. Cette crainte est donc à écarter définitivement.

\section{2) Conditions relatives à l'hygiène - Absence de germes pathogènes}

Nous avons volontairement laissé de côté jusqu'ici cette notion, cependant nécessaire, de l'absence de germes pathogènes dans le lait stérilisé. La raison en est simple : l'absence indispensable de micro-organismes susceptibles de compromettre la santé des consommateurs est une notion très générale, intéressant tous les aliments. Elle n'est pas spéciale au lait stérilisé ; elle n'en constitue pas un caractère spécifique. En disant que, comme tous les aliments, le lait stérilisé doit être exempt de germes pathogènes, comme il doit être loyal et marchand, on n'ajoute rien à sa définition.

Cependant, lorsque nous disons que « le lait stérilisé " doit être exempt de micro-organismes susceptibles d'y proliférer, nous ne sommes pas à l'abri de la présence éventuelle de germes pathogènes ou de toxines. En effet :

- Le lait stérilisé peut renfermer des spores de germes pathogènes, non susceptibles de proliférer, sans cesser d'être conforme aux éléments de définition déjà donnés plus haut.

- Même observation en ce qui concerne la présence éventuelle de germes toxinogènes.

- Il pourrait également contenir des toxines thermo-résistantes dont l'absence n'a pas été prescrite jusqu'ici.

Donc, de toute manière, dans un lait stérilisé conforme aux conditions déjà exprimées, la présence de micro-organismes nuisibles ou de leurs toxines reste possible. Or, elle est indésirable et aucune définition contrôlable ne saurait postuler leur absence. Donc nous devons envisager une définition plus large, impliquant cette condition supplémentaire. 


\section{3) Durée de conservation des laits stérilisés}

On serait tenté de souhaiter (et de croire possible) une conservabilité indéfinie du lait stérilisé. Outre que cela serait pratiquement inutile, il convient de montrer que cela est impossible pour des raisons qui n'ont rien à voir avec la bactériologie.

En effet :

Dans un lait qui serait stérile (au sens absolu) des modifications de nature physico-chimique interviendraient inévitablement avec le temps. L'émulsion grasse, malgré l'homogénéisation qui diminue la force ascensionnelle des globules gras, sans l'annuler complètement, se déstabilise progressivement surtout si la température ambiante s'élève et diminue la viscosité du produit. Il n'y a pas rupture de l'émulsion, mais seulement crémage spontané dont la vitesse est fonction du temps et de la température.

Dans les laits stérilisés non soumis à la désaération préalable, des phénomènes d'altération oxydative de la matière grasse peuvent aussi intervenir, plus ou moins rapidement, causant un suiffage parfois suivi d'un rancissement qui rendent, à la longue, le produit inconsommable. L'action de la lumière dans le cas des récipients de verre, accélère évidemment ces phénomènes.

D'autre part, la phase colloïdale tend également à se déstabiliser sous l'action de la pesanteur et, dans les laits les plus stables, obtenus grâce aux techniques actuelles, on parvient à constater, après un long temps de repos, la formation d'un dépôt de phosphocaséinate de calcium au fond des récipients.

Ces trois phénomènes, dans les laits de bonne qualité, sont extrêmement lents et peuvent n'apparaître qu'au bout de plusieurs années. Mais ils aboutissent à rendre le lait stérilisé non marchand. Dans la pratique, leur extrême lenteur est le plus souvent sans inconvénient. Mais, à eux seuls, ils s'opposent formellement à l'introduction, dans la définition du lait stérilisé, de la notion de conservation indéfinie.

\section{4) Conditions relatives aux récipients}

Quelle que soit la technique de stérilisation (en récipients ou en flux continu suivie du remplissage aseptique en récipients stériles), le lait stérilisé doit être parfaitement à l'abri de toute contamination et de toute altération pendant son stockage, son transport et sa mise en vente.

Cela signifie que les récipients qui renferment le lait stérilisé doivent être parfaitement étanches non seulement aux micro-organismes mais aussi aux liquides et aux gaz.

Enfin, il serait souhaitable que ces récipients fussent opaques pour protéger le lait stérilisé contre l'action de la lumière. A défaut 
de cette condition, il doit être spécifié que le lait stérilisé ne peut être entreposé qu'à l'abri de la lumière.

Nous possédons maintenant tous les éléments nous permettant de définir correctement, à notre avis, le lait stérilisé. Nous proposons donc la définition suivante :

\section{DEFINITION DU LAIT STERILISE}

Le lait stérilisé est un lait qui a été traité par chauffage (ou par tout autre procédé officiellement autorisé) de telle manière qu'il satisfasse aux conditions suivantes:

1) Etre exempt de micro-organismes nuisibles à la santé des consommateurs, c'est-à-dire ne pas renfermer de germes pathogènes ou toxinogènes.

2) Etre exempt de micro-organismes quelconques susceptibles de s'y développer, c'est-à-dire n'être le siège d'aucune prolifération.

3) Etre et rester parfaitement stable et de bonne qualité organoleptique, c'est-à-dire ne présenter aucun signe d'altération pendant une période assez longue, compatible avec les nécessités commerciales.

4) Ne pas renfermer, après trois semaines d'incubation à $31^{\circ} \mathrm{C}$ $\pm 1^{\circ} \mathrm{C}$, plus de 5 spores vivantes par $m l$, incapables de proliférer dans le lait, révélées par culture sur plaques dans un milieu nutritif approprié.

5) Le lait stérilisé doit être conditionné dans des récipients étanches aux liquides, aux gaz et aux micro-organismes.

6) Le lait stérilisé doit être entreposé à l'abri de la lumière ou conditionné en récipients opaques.

Désignation : La désignation officielle d'un tel lait ne peut être que "lait stérilisé ".

\section{CAS PARTICULIER DES LAITS U.H.T.}

On sait qu'il existe maintenant plusieurs techniques de stérilisation du lait :

1) Stérilisation en récipients, après conditionnement et bouchage hermétique, qui se subdivise en deux groupes :

a) Stérilisation discontinue, en autoclaves classiques, qui groupe tous les procédés traditionnels, 
b) Stérilisation continue, en autoclaves à colonnes de pression d'eau alimentés en continu.

2) Stérilisation en flux continu, suivie du conditionnement aseptique en récipients stériles, appelée encore stérilisation U.H.T.

On connaît les différences qui séparent ces deux groupes de méthodes :

\section{a) CAS DE LA STÉRILISATION EN RÉCIPIENTS}

Dans le cas de la stérilisation en récipients (même agités) les échanges thermiques ne sont pas instantanés et, pour obtenir le résultat cherché (c'est-à-dire une très bonne conservation du lait stérilisé au cours d'un stockage de longue durée à la température ordinaire) il est indispensable de prolonger la période de chauffage (à $115^{\circ} \mathrm{C}$ ou $120^{\circ} \mathrm{C}$ par exemple) pendant une durée égale ou supérieure à $15-20 \mathrm{mn}$.

La pratique s'accorde avec la théorie pour admettre que, dans ces conditions, les bouteilles de lait ainsi traité ne sont pas toutes stériles et, pour les raisons indiquées plus haut, un certain nombre d'entre elles (toujours inconnu et imprévisible) peuvent renfermer des spores vivantes incapables de germer et de proliférer dans le lait. En outre, tous les laits traités par ces techniques présentent une couleur et une saveur légèrement modifiées, anormales, parfois désagréables.

\section{b) CAS DE LA STÉRILISATION U.H.T.}

En revanche, dans la technique des laits U.H.T., le chauffage en flux continu, c'est-à-dire sans l'interposition de la paroi des récipients entre le fluide chauffant et le fluide chauffé, c'est-à-dire encore, comportant des échanges thermiques instantanés et excellents, les conditions de la stérilisation sont modifiées : il est possible d'utiliser des températures très élevées (U.H.T. c'est-à-dire ultra-haute température) pendant des temps très courts car l'efficacité bactéricide de la chaleur est beaucoup plus fonction de la température que de la durée d'application de cette température. Ce que nous appelons le $\mathrm{Q}_{10}$ de la destruction des spores, c'est-à-dire la variation de la vitesse de cette réaction bactéricide pour une élévation de température de $10^{\circ} \mathrm{C}$, est importante, de l'ordre de 8 à 10 . Cette augmentation quasi-logarithmique de l'efficacité stérilisante quand la température passe de $120^{\circ} \mathrm{C}$, à $130^{\circ} \mathrm{C}$, à $140^{\circ} \mathrm{C}$, à $150^{\circ} \mathrm{C}$ (et qui varie à peu près comme $1,10,100,1000$ ) permet donc d'avoir les mêmes résultats avec des durées d'application variant en sens inverse comme $1000,100,10,1$, ce qui signifie que si, dans des conditions données, il faut appliquer la température de $120^{\circ} \mathrm{C}$ pendant $2000 \mathrm{~s}$ (par exemple), le même résultat bactériologique sera obtenu à $150^{\circ} \mathrm{C}$ en $2 \mathrm{~s}$. 


\section{1) Caractères physiques du lait U.H.T.}

Or le $\mathrm{Q}_{10}$ des réactions chimiques a une valeur beaucoup moindre (3 à 4 fois) que celui des réactions bactéricides et, d'autre part, ces réactions chimiques (changement de coloration, de saveur, etc.) présentent une intensité proportionnelle à la durée d'application de la température. Pour ces deux raisons, l'élévation de la température, dans les conditions d'application de la stérilisation U.H.T., se traduit par des modifications chimiques de plus en plus faibles au fur et à mesure que les températures sont plus élevées et les durées d'application plus courtes (Burton [4], Galesloot [10], Pien [18]).

La conclusion pratique de ce qui précède est la suivante : le lait stérilisé U.H.T. (par exemple à $150^{\circ} \mathrm{C}$ pendant $2 \mathrm{~s}$ ) ne présente aucun des défauts de coloration, saveur, etc. du lait stérilisé dans les conditions classiques (par exemple à $120^{\circ} \mathrm{C}$ pendant $15-20 \mathrm{mn}$ ). II est blanc et de saveur normale.

\section{2) Caractères bactériologiques du lait U.H.T.}

Qu'en est-il du degré de stérilité des laits U.H.T. ?

Certains auteurs (et surtout certains hygiénistes, non spécialistes de la technologie laitière) ont prétendu que le lait U.H.T. était stérile.

Or le problème se pose exactement comme dans le cas de la stérilisation classique et pour les mêmes raisons : le lait cru contient ou peut contenir, en quantités variables mais toujours faibles, des spores très thermorésistantes qui ne sont pas détruites par les conditions de la stérilisation classique ni par celles de la stérilisation U.H.T., (dont certaines ne peuvent pas germer, ni par conséquent proliférer, dans les conditions normales du stockage des laits stérilisés). Il se trouve donc que certains récipients de lait stérilisé (classique ou U.H.T.) peuvent renfermer une ou plusieurs spores ayant survécu au traitement thermique. Donc, le contenu de ces récipients n'est pas stérile, mais nous savons que cela est sans importance si ces spores ne peuvent pas se développer, donc altérer le lait. Tout ce qui a été dit plus haut retrouve sa place ici, dans le cas des laits U.H.T. et pour les mêmes raisons.

C'est pourquoi il faut conclure que la stérilité (dont nous avons montré qu'elle n'était pas nécessaire) est aussi impossible à obtenir d'une manière générale et absolue dans la préparation des laits U.H.T. que dans celle des laits stérilisés classiques et, par conséquent, il ne faut pas prétendre que de tels laits sont " stériles ", même si un pourcentage élevé de récipients le sont.

Des preuves directes de cette affirmation ont été données par divers auteurs qui ont volontairement contaminé du lait cru avec des spores de germes très thermorésistants, ont soumis ce Iait à des traitements U.H.T. et ont retrouvé dans le lait ainsi traité, des quan- 
tités de spores vivantes, variables certes, mais jamais nulles, même dans de nombreux cas, en l'absence de toute altération du lait après incubation $[1,2,3,6$ à $9,13,15,16,22,23]$.

\section{3) Remplissage aseptique du lait U.H.T.}

Nous n'avons pas parlé du remplissage aseptique des laits U.H.T. parce que chacun sait que les chauffages très brefs à très haute température dont il a été parlé ci-dessus ne peuvent pas être réalisés dans le lait préalablement réparti dans des récipients clos. Cette condition ne peut être satisfaite, pour des raisons évidentes, que sur le lait liquide circulant en flux continu dans des appareils appropriés. Ce lait stérilisé doit donc obligatoirement être introduit aseptiquement dans des récipients stériles et c'est précisément ce qui se fait tous les jours dans les usines appliquant les principes du traitement U.H.T.

Si le lait chauffé à ultra-haute température (par exemple stérilisé $2 \mathrm{~s}$ à $150^{\circ} \mathrm{C}$ ou $10-20 \mathrm{~s}$ à $140^{\circ} \mathrm{C}$ ) n'était pas conditionné aseptiquement il serait contaminé plus ou moins gravement et sa conservabilité, voire sa qualité hygiénique, seraient compromises (ce que l'expérience a confirmé).

\section{4) Elaboration de la définition du lait U.H.T.}

Il apparaît clairement, à la suite de ce qui vient d'être dit, que le lait U.H.T. n'est pas plus un lait "stérile »que le lait stérilisé traditionnel. Mais, sa probabilité de stérilité n'est pas moindre et, du point de vue bactériologique et commercial (conservation) ces deux types de laits sont identiques et doivent comporter la même désignation qui ne peut être que celle de lait stérilisé.

En quoi consiste donc la supériorité du lait stérilisé U.H.T. sur le lait stérilisé traditionnel ?

Nous l'avons dit plus haut : les caractères organoleptiques du lait U.H.T. (couleur, saveur, etc.) sont très supérieurs à ceux du lait stérilisé classique.

Nous en avons donné les raisons. Il serait donc souhaitable que cette supériorité, réelle et très importante, fût indiquée dans la définition. Or, il existe un moyen permettant de distinguer ces deux sortes de laits, en dehors de l'application subjective des caractères organoleptiques : la considération du $\mathrm{Q}_{10}$ des réactions chimiques par rapport à celui des réactions bactéricides et la possibilité de chauffer pendant des temps très courts à très haute température, amenuise de façon considérable l'intensité des réactions chimiques (et physico-chimiques). En dehors de la couleur et de la saveur, nous savons maintenant que l'insolubilisation de l'albumine du lait, totale dans la stérilisation classique, est faible ou très faible dans les stérilisations U.H.T. 
Donc, pour qu'un lait stérilisé U.H.T. puisse se prévaloir de cette dénomination, il faut que ce caractère soit vérifié. Une telle distinction est maintenant parfaitement possible grâce à la réaction d'Aschaffenburg (1) bien connue des spécialistes, que nous avons (à la demande de la Fédération Internationale de Laiterie) adaptée à ce problème particulier. Le Groupe d'Experts de la F.I.L. chargé de l'étude des laits U.H.T. a adopté nos propositions et décidé de désigner cette réaction modifiée sous le nom de test de turbidité.

En conclusion le "test de turbidité », appliqué aux laits stérilisés U.H.T., doit donner une réponse positive (présence d'albumine soluble).

Remarque: On pourrait aller plus loin et proposer d'introduire également ce test dans la définition des laits stérilisés classiques (où il doit donner une réponse négative). Nous ne pensons pas que cela soit indispensable, car si le lait stérilisé est, comme il se doit à notre avis, conforme à la définition générale donnée plus haut, ce résultat peut être obtenu de deux façons : soit par les techniques de traitement U.H.T. qui conduisent à un test de turbidité positif, soit autrement (c'est-à-dire par les techniques traditionnelles de stérilisation en récipients) et, dans ce cas, le test de turbidité est négatif. On pourrait, certes, admettre qu'une stérilisation " classique » conduise à un test de turbidité plus ou moins positif, ce qui correspondrait à l'application de températures beaucoup trop basses ou à des durées d'application beaucoup trop courtes. Or, dans ce cas, les caractères exigés de tout lait stérilisé dans la définition générale ne seraient pas respectés et un tel lait stérilisé ne serait pas acceptable. Il n'aurait pas droit à la dénomination « lait stérilisé ». Par conséquent, il n'y a aucun-risque d'équivoque à ne pas faire figurer cette mention du test de stabilité dans la définition générale du lait stérilisé.

D'ailleurs, il est facile de comprendre que ce test ne pourrait pas figurer dans la définition générale qui couvre tous les laits stérilisés, aussi bien les laits U.H.T. (où le test doit être positif) que les laits stérilisés classiques (où le test doit être négatif). Ce n'est que dans une définition particulière aux laits stérilisés classiques que l'obligation de prévoir un test de turbidité négatif pourrait se concevoir, dans le but, par exemple, de simplifier les opérations de contrôle.

(1) Cette réaction était utilisée, jusqu'ici, à la distinction des laits pasteurisés (où l'albumine est en grande partie non dénaturée et décelable) et des laits stérilisés classiques (où l'albumine est totalement dénaturée). Or on a depuis longtemps constaté que cette réaction était inutilisable pour distinguer les laits pasteurisés et les laits stérilisés U.H.T., car dans les deux cas, elle donne une réaction positive d'albumine non dénaturée. C'est la raison qui nous a incité à utiliser cette réaction (en la modifiant pour l'adapter) afin d'effectuer la distinction entre les laits U.H.T. (où une grande partie de la lactalbumine reste soluble) et les laits stérilisés classiques (où l'albumine est entièrement dénaturée). 
Nous possédons maintenant tous les éléments d'une définition correcte du lait U.H.T.

\section{DEFINITION DU LAIT U.H.T.}

Le lait U.H.T. est un lait stérilisé obtenu dans les conditions suivantes :

1) Chauffage en flux continu du lait non conditionné.

2) Chauffage direct du lait cru (donc aucun chauffage avant le traitement U.H.T.).

3) Chauffage à haute température (au moins $135^{\circ} \mathrm{C}$ ) pendant un temps très court (au maximum quelques secondes aux plus hautes températures et moins de $1 \mathrm{mn}$ aux températures les plus basses) suivi d'homogénéisation et de refroidissement aseptiques immédiats.

4) Chauffage suivi de conditionnement aseptique en récipients stériles.

5) Si le conditionnement ne suit pas immédiatement la stérilisation, le lait stérilisé refroidi doit être stocké aseptiquement en réservoirs stériles sans aucun risque de contamination. La durée de ce stockage ne doit pas excéder $24 \mathrm{~h}$.

6) Le lait U.H.T. étant un lait stérilisé doit répondre aux conditions requises dans la définition générale du lait stérilisé et satisfaire aux normes de contrôle définies plus loin.

7) Le lait U.H.T. doit donner une réponse positive lors de l'application du test de turbidité (voir plus loin).

Désignation : La désignation officielle d'un tel lait doit être " lait stérilisé U.H.T. »ou, simplement, " lait U.H.T. ".

Remarques relatives à l'appellation " lait stérilisé ": Certains spécialistes estiment que le mot " lait stérilisé » ne peut signifier que "lait stérile " et pensent que cette dernière expression devrait remplacer la précédente.

Or, nous avons vu plus haut que le lait stérile est une entité théorique, irréalisable au sens absolu dans la pratique, incontrôlable en tout cas, car la stérilité ne peut pas être démontrée et, par dessus tout, parfaitement inutile puisque l'on peut obtenir des laits de très longue conservation, exempts de germes dangereux et de germes d'altération, et impossibles à distinguer de laits qui seraient absolument stériles.

Donc, l'expression lait stérile n'est pas défendable et n'est pas utilisable car elle n'a pas de signification pratique. Les laits soumis à l'opération de stérilisation dans des conditions qui les rendent conformes aux définitions données plus haut (c'est-à-dire satisfai- 
sants à tous égards) ne sont pas nécessairement stériles. Ils ont cependant été "stérilisés " et c'est cette dénomination qui doit permettre de les désigner et de les définir, comme ayant subi la stérilisation (au même titre que les laits pasteurisés sont des laits qui ont subi la pasteurisation).

D'autre part, l'usage international tout à fait général des mots " lait stérilisé » doit être respecté. Nous ne devons pas, en France, méconnaître cette position et nous en écarter. Nous ne nous ferions plus comprendre dans le commerce international. Or le lait stérilisé fait en France, l'objet d'exportations de plus en plus importantes qui risqueraient fort d'être compromises si nous changions l'appellation.

Les organisations internationales (F.A.O., O.M.S., etc.) utilisent l'expression "lait stérilisé " dans le sens que nous lui donnons ici.

Le Marché Commun reconnaît également cette expression et les règles qui seront établies pour des échanges internationaux concernant ce produit ne s'appliqueront évidemment pas à un produit portant un autre nom.

D'ailleurs dans tous les pays étrangers l'expression « lait stérilisé » existe et continue d'être régulièrement utilisée.

Serions-nous amenés à ne plus exporter de lait stérilisé sous le prétexte qu'aucun produit répondant à cette définition internationale n'existerait plus en France? Il y a là quelque chose de très grave.

Conclusion : Pour désigner le lait stérilisé, tel que nous l'avons défini plus haut, il faut, d'une part, s'interdire l'utilisation du terme " lait stérile " qui, on l'a vu, n'a pas de signification précise, et, d'autre part, renoncer à toute autre expression que celle de "lait stérilisé » qui donne entière satisfaction, qui est utilisée dans le monde entier et désigne avec certitude le produit qui a été défini plus haut.

 \\ DEUXIÈME PARTIE \\ CONTROLE DU LAIT STERILISE}

La Fédération Internationale de Laiterie a longuement étudié cette question. Elle a constitué un Groupe d'Experts (1) qui, après

(1) Composition du Groupe d'Experts chargé de l'étude du contrôle du lait stérilisé : MM. Borgeaud (CH), Demeter (B), Franklin (UK), Galesloot (NL), Mocquot (F), Negri (I), Pien (F) responsable du Groupe et rapporteur, Samuelsson $(\mathrm{S})$. 
plusieurs années d'étude, a mis au point un document important, publié comme Norme Internationale de la F.I.L. ( $\left.n^{\circ} 48,1969\right)$.

Les principes qui ont guidé les Experts dans leur travail ont été les suivants :

1) Le lait stérilisé devant être, conformément à ce qu'on en attend, parfaitement stable au cours d'une conservation prolongée, les épreuves doivent essentiellement porter sur le contrôle de la stabilité physico-chimique du lait stérilisé soumis à des incubations appropriées.

2) Le lait stérilisé ne devant être le siège d'aucun développement microbien, mais pouvant néanmoins renfermer quelques rares microorganismes ayant survécu à la stérilisation et incapables de se développer dans le lait, il est procédé à un examen bactériologique sommaire (qui ne doit laisser apparaître, au cours de cultures sur milieux solides, qu'un très faible nombre de colonies).

L'application des principes donne lieu, de notre part, aux commentaires suivants :

\section{1) Concernant la stabilité physico-chimique du lait}

Nous avons admis que le lait est stable si, après incubation à $30^{\circ} \mathrm{C}$ et à $55^{\circ} \mathrm{C}$ :

- Ie test à l'alcool n'entraîne aucune floculation,

- l'acidité, après incubation, n'est pas différente de plus de $0,2 \mathrm{~g}$ exprimée en acide lactique par litre, de ce qu'elle était avant incubation,

- le $\mathrm{pH}$, après incubation, n'est pas différent de plus de 0,5 unité de ce qu'il était avant incubation,

- l'examen organoleptique ne révèle pas, après incubation, de modifications anormales de l'aspect, de la couleur, de la saveur (en dehors de l'évolution que certaines de ces caractéristiques subissent normalement au cours d'un séjour prolongé à l'étuve).

\section{2) Concernant la stabilité microbiologique du lait}

L'absence de prolifération de micro-organismes (quels qu'ils soient) au cours des incubations est mise en évidence par un examen microscopique direct d'une petite quantité de lait étalée sur lame. Cet examen, effectué avant et après incubation, doit donner des résultats comparables (voir plus loin le mode opératoire proposé).

D'autre part nous avons admis que le lait stérilisé pouvait renfermer quelques spores capables de survivre à la stérilisation, mais que le nombre devait en être très limité. Les avis diffèrent quant à ce nombre limite acceptable. Mais, de toute façon, il ne peut s'agir que de spores incapables de germer, donc de proliférer, au cours de l'incubation à laquelle sont soumis les échantillons. Par conséquent, 
en procédant à cet examen après incubation et en constatant que le nombre de germes vivants (colonies sur milieu gélosé) est très petit, on aura la preuve que le lait remplit à la fois les deux conditions imposées. (Si l'on avait affaire à des spores survivantes capables de germer et de proliférer, le nombre de germes ainsi formés au cours de l'incubation serait considérable et l'on en serait déjà averti par l'examen microscopique direct, éventuellement par l'altération du lait, rendant ainsi inutile ce contrôle par culture).

Ce n'est donc que si les autres tests donnent, après incubation, des résultats conformes à la définition du lait stérilisé, que l'on envisagera de procéder à cette épreuve complémentaire, uniquement destinée à dépister les « spores dormantes » et à n'accepter pour "bons " que les laits stérilisés n'en refermant qu'un très petit nombre. (Les spécialistes français ont admis, depuis longtemps, que ce nombre, avant ou après incubation, ne devait pas dépasser 5 par ml. Une disposition analogue figure déjà dans la réglementation française (arrêté du 13 août 1963) concernant les laits concentrés non sucrés stérilisés). La norme no 48 de la F.I.L. en tolère jusqu'à 100 par $\mathrm{ml}$ (10 par inoculum de $0,1 \mathrm{ml})$. Ce chiffre semble très élevé à certains spécialistes. En fait, dans la pratique, il est toujours très loin d'être atteint.

Les conditions à remplir, selon nous, par le lait stérilisé ont été précisées plus haut à l'occasion de la définition que nous avons proposée du lait stérilisé.

Quant à la méthode de contrôle elle-même, nous proposons l'adoption de la norme F.I.L. no 48 légèrement modifiée pour tenir compte de la norme française déjà existante relative au contrôle des laits concentrés non sucrés (arrêté du 13 août 1963, annexe II) et des indications données par les Professeurs G. Thieulin et R. Vuillaume dans leur excellent Manuel d'Analyse et d'Inspection du lait [22] et par celles données également par le Professeur Buttiaux dans la Monographie F.A.O., $\mathrm{n}^{\circ}$ 65, sur la stérilisation du lait [5].

La combinaison de ces quatre documents conduit au texte suivant dont nous proposons l'adoption :

\section{METHODE DE CONTROLE DU LAIT STERILISE (projet)}

Le contrôle bactériologique des laits stérilisés a pour but de rechercher si ces laits ont subi un traitement convenable et suffisant pour assurer l'absence de germes pathogènes et la stabilité de la qualité marchande (1).

(1) Le contrôle des laits U.H.T. (qui sont des laits stérilisés) s'effectuera par cette méthode, complétée par le test de turbidité, décrit plus loin. 
Il nécessite, pour chaque contrôle, le prélèvement de cinq échantillons.

\section{a) Examen avant incubation}

Sur un échantillon non incubé sont effectués :

- Un examen organoleptique (aspect, odeur, saveur).

- Une mesure électrométrique du $\mathrm{pH}$ et de l'acidité titrable.

- Un examen bactériologique (numération directe approximative sous le microscope) (1).

\section{b) Epreuve d'étuvage}

Deux autres échantillons sont mis à incuber à $30^{\circ} \mathrm{C} \pm 1^{\circ} \mathrm{C}$ pendant $14 \mathrm{j}$. Après incubation, l'un d'eux servira aux examens organoleptique, physique et bactériologique décrits au paragraphe c, l'autre sera réservé à l'examen bactériologique (dénombrement par culture) décrit au paragraphe $d$.

Les deux derniers échantillons sont mis à incuber à $55^{\circ} \mathrm{C} \pm 1^{\circ} \mathrm{C}$ pendant $7 \mathrm{j}$. Après incubation, ils subiront respectivement les mêmes examens.

c) Examens organoleptique, physique et bactériologique après incubation.

Le produit doit satisfaire simultanément à toutes les épreuves suivantes :

\section{1) Examen organoleptique}

Examen visuel en vue de déceler toute trace de coagulation ou de protéolyse (si elle n'est pas apparente, centrifuger, par exemple $5 \mathrm{mn}$ à 1000 tours $/ \mathrm{mn}$, pour accélérer la sédimentation éventuelle).

(1) Nous recommandons tout particulièrement la méthode suivante que nous avons mise au point jadis (Le Lait, juillet-août 1931, p. 705 à 711) et qui nous a toujours donné d'excellents résultats :

1) $5 \mathrm{~cm}^{3}$ de lait sont additionnés de $1 \mathrm{~cm}^{3}$ d'une solution de chlorure de calcium cristallisé à 50 p. 100 dans l'eau (dans le but de régulariser le séchage).

2) Etaler $1 / 100$ de $\mathrm{cm}^{3}$ de ce mélange sur un carré de $1 \mathrm{~cm}$ de côté, tracé au diamant sur une lame de verre.

3) Sécher, laver à l'alcool à $90^{\circ} \mathrm{C}$ (pour éliminer le $\mathrm{CaCl}_{2}$ ), sécher, dégraisser au xylol, rincer à l'éther, sécher, colorer au bleu de méthylène, rincer à l'eau, sécher.

4) Examiner à l'aide d'un objectif à immersion, en ayant soin de jauger le champ pour qu'il ait un diamètre de $120 \mu(0,12 \mathrm{~mm})$.

5) Explorer une ou plusieurs bandes d'un trait de diamant à l'autre (c'està-dire des bandes de $1 \mathrm{~cm}$ de long et de $120 \mu$ de large).

Un germe rencontré dans une de ces bandes correspond à 10000 germes dans $1 \mathrm{~cm}^{3}$ de lait initial. 
L'odeur et la saveur de l'échantillon ne doivent pas être différentes de celles d'un lait stérile soumis à une incubation prolongée à l'étuve.

\section{2) Epreuve à l'alcool}

Mélanger à parties égales : lait à étudier et alcool éthylique à $68^{\circ}$. Il ne doit se produire aucune floculation.

\section{3) Epreuve d'ébullition}

Introduire quelques $\mathrm{cm}^{3}$ de lait stérilisé dans un tube à essai et porter $5 \mathrm{mn}$ au BM bouillant. Il ne doit se produire aucune floculation.

\section{4) Mesure électrométrique du $\mathrm{pH}$}

Le $\mathrm{pH}$ du lait stérilisé mesuré après incubation ne doit pas différer du $\mathrm{pH}$ mesuré avant incubation de plus de 0,5 unité.

\section{5) Mesure de l'acidité titrable}

L'acidité titrable du lait stérilisé mesurée après incubation ne doit pas différer de l'acidité titrable mesurée avant incubation de plus de 0,2 exprimée en grammes d'acide lactique par litre de lait.

\section{6) Examen bactériologique}

L'examen microscopique direct (effectué par la méthode directe décrite plus haut) ne doit pas faire apparaître un nombre de germes totaux sensiblement plus élevé que dans le cas de l'examen avant incubation.

d) Examen bactériologique (Dénombrement par culture des formes revivifiables).

Le cas échéant, on procèdera aux épreuves suivantes :

1) En aérobiose

Un $\mathrm{ml}$ du contenu de l'échantillon est placé aseptiquement dans une boîte de Pétri de $100 \mathrm{~mm}$ de diamètre. On le mélange de façon très homogène avec $20 \mathrm{ml}$ du milieu stérilisé suivant :

Peptone trypsique de caséine (Bacto-tryptone Difco no B 123) $\ldots \ldots \ldots \ldots \ldots$

Extrait de levure $\ldots \ldots \ldots \ldots \ldots$



Amidon soluble (exempt de propriétés bactéricides)

Agar . . . . . . . . . . . . . . . . . . . . . . . . . . . . . . .

Eau distillée dans un appareil en verre ou en silice

Ajuster à $\mathrm{pH}$ : 7. Répartir le milieu à raison de $20 \mathrm{ml}$ par tube de $200 \times 20 \mathrm{~mm}$. Autoclaver $20 \mathrm{mn}$ à $115^{\circ} \mathrm{C}$. Conserver au frais et à l'obscurité. 
Ce milieu est fondu préalablement au bain-marie bouillant puis ramené à la température de $48^{\circ} \mathrm{C}$.

Le milieu ainsi ensemencé est incubé pendant 3 j à $31^{\circ} \mathrm{C} \pm$ $1^{\circ} \mathrm{C}$ ou à $55^{\circ} \mathrm{C} \pm 1^{\circ} \mathrm{C}$ selon que l'échantillon a été incubé lui-même à l'une ou à l'autre de ces températures.

\section{2) En anaérobiose}

Un $\mathrm{ml}$ du contenu de l'échantillon est introduit aseptiquement dans $20 \mathrm{ml}$ du milieu V.L. (viande extrait de levure) ci-après :

Peptone trypsique de caséine (Bacto-tryptone Difco

$\mathrm{n}^{\mathrm{o}}$ B 123) . . . . . . . . . . . . . . . . . . . . . .

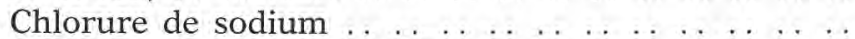

Extrait de viande (Liebig) . . . . . . . . . . . . . . . . . . .

Extrait de levure . . . . . . . . . . . . . . . . . . . . . . .

Glucose . . . . . . . . . . . . . . . . . . . . $\ldots \ldots \ldots \ldots$

Chlorhydrate de cystéine ... . . . . . . . . . . . . . . .

Amidon soluble (exempt de propriétés bactéricides)

\section{Agar}

Eau distillée dans un appareil en verre ou en silice

Faire bouillir sans le glucose. Ajuster à pH 7,4. Répartir le milieu à raison de $20 \mathrm{ml}$ par tube de $200 \times 20 \mathrm{~mm}$. Autoclaver $20 \mathrm{mn}$ à $115^{\circ} \mathrm{C}$. Conserver au frais et à l'obscurité.

On opère ainsi :

Le tube de $200 \times 20 \mathrm{~mm}$ contenant le milieu en culot est placé au bain-marie bouillant; après fusion, il y reste durant $10 \mathrm{mn}$ au moins pour éliminer parfaitement les gaz dissous. La température du milieu est alors ramenée à $48^{\circ} \mathrm{C}$. On ajoute le lait et on mélange de façon homogène en évitant toute agitation excessive capable d'introduire une trop grande quantité d'air. On refroidit sous l'eau du robinet et on laisse gélifier en culot.

Le milieu ainsi ensemencé est incubé pendant $3 \mathrm{j}$ à $31^{\circ} \mathrm{C} \pm 1^{\circ} \mathrm{C}$ ou à $55^{\circ} \mathrm{C} \pm 1^{\circ} \mathrm{C}$ selon que l'échantillon a été incubé lui-même à l'une ou à l'autre de ces températures.

On considère comme acceptables les produits ne contenant pas plus de 5 bactéries par $\mathrm{ml}$, c'est-à-dire pas plus de 5 colonies sur chacun des deux milieux.

e) Test de turbidité (Distinction des laits U.H.T. et des laits stérilisés traditionnels).

Nous avons dit plus haut (Définition du lait U.H.T.) que les laits stérilisés en flux continu à haute température pendant un temps très court devaient renfermer une certaine quantité d'albumine soluble alors que les laits stérilisés classiques (stérilisation en récipients à des températures de l'ordre de $120^{\circ} \mathrm{C}$ pendant plusieurs minutes) ne devaient pas renfermer d'albumine soluble. 
L'épreuve qui permet de distinguer ces deux types de laits stérilisés est appelée « test de turbidité ». Elle est obligatoire dans le cas des laits U.H.T. puisqu'elle fait partie de la définition proposée de ces laits. Elle ne l'est pas dans le cas des laits stérilisés classiques, parce que, si toutes les autres conditions exigées sont remplies (stabilité physique, absence de développement microbien au cours de l'incubation, etc.) le test de turbidité sera nécessairement négatif. Cependant on pourrait fort bien concevoir que cette épreuve fût également rendue obligatoire dans le but d'obtenir une garantie supplémentaire ou pour rendre le contrôle plus rapide et plus simple.

Le texte de cette épreuve, fondé sur le test d'Aschaffenburg, a été mis au point par nos soins à la demande du Groupe d'Experts de la Fédération Internationale de Laiterie (1) chargé de l'étude des laits U.H.T.

Nous reprendrons donc, purement et simplement, le texte adopté par la F.I.L. (Melbourne 1970, Comm. B, Doc. 2, Annexe 1) qui est le suivant :

\section{METHODE PERMETTANT DE DISTINGUER LES LAITS U.H.T. DES AUTRES LAITS STERILISES}

(test de turbidité modifié)

\section{1) Domaine d'application}

L'objet du test décrit ci-dessous est de permettre la distinction entre les laits U.H.T. (c'est-à-dire des laits stérilisés obtenus par un procédé de chauffage en flux continu pendant quelques secondes à une température supérieure à $130^{\circ} \mathrm{C}$ ), des autres types de laits stérilisés suivant des méthodes classiques (stérilisation dans les récipients par systèmes statiques ou rotatifs pendant plusieurs minutes ou davantage à des températures inférieures à $125^{\circ} \mathrm{C}$ ).

La méthode est applicable à l'identification de laits U.H.T. aromatisés ou chocolatés qui n'ont pas été soumis à un pré-chauffage.

\section{2) Principe de la méthode}

Le test de turbidité consiste à déterminer la présence éventuelle de protéines de sérum non dénaturées dans un lait stérilisé. Cette détermination est basée sur l'aspect visuel après $5 \mathrm{mn}$ d'ébullition du filtrat obtenu à partir d'un échantillon de lait auquel on a ajouté

(1) Composition du Groupe d'Experts : MM. Ashton (GB) rapporteur, Drogt (NL), Haukka (SF), Hostettler (CH), Lembke (D), Negri (I), Pien (F), Ronkilde-Poulsen (DK), Samuelsson (S), Shew (AUS), O'Sullivan (EIR). 
une quantité donnée de sulfate d'ammonium. Lorsqu'il est soumis à ce traitement, le lait contenant des protéines de sérum non dénaturées présentera une turbidité positive ; réciproquement, une turbidité négative indique l'absence de protéines de sérum non dénaturées.

Les protéines de sérum non dénaturées résiduelles sont présentes en quantités négligeables ou sont absentes dans le lait stérilisé suivant les méthodes classiques et ce lait présente une absence de turbidité ou une réaction négative. Le lait U.H.T., cependant, contient des quantités appréciables de protéines de sérum non dénaturées et accuse invariablement la présence de turbidité, ou une réaction positive.

\section{3) Réactifs}

3.1. Sulfate d'ammonium : $\left(\mathrm{NH}_{4}\right)_{2} \mathrm{SO}_{4}$.

\section{4) Appareillage}

4.1. Béchers de $100 \mathrm{ml}$ ou flacons coniques de $100 \mathrm{ml}$.

4.2. Entonnoirs et papier filtre (à filtration lente - Whatman $n^{\circ}$ $2 \mathrm{~V}$ ou 42).

4.3. Tubes à essai $160 \times 16 \mathrm{~mm}$.

4.4. Bain-marie contenant de l'eau en ébullition au moment où les tubes y sont plongés ; l'eau devra rester en ébullition jusqu'à la fin du test.

\section{5) Mode opératoire}

5.1. Verser dans un bécher ou un flacon conique, à l'aide d'une pipette graduée, $20 \mathrm{ml} \pm 0,5 \mathrm{ml}$ de l'échantillon de lait rendu homogène par retournement ou agitation.

5.2. Ajouter $4 \pm 0,1 \mathrm{~g}$ de sulfate d'ammonium. Agiter doucement le bécher ou le flacon pendant $1 \mathrm{mn}$ et laisser reposer pendant $4 \mathrm{mn}$.

5.3. Filtrer à travers du papier filtre (4.2). Verser $5 \mathrm{ml} \mathrm{du}$ filtrat dans un tube à essai (4.3). Si nécessaire, filtrer à nouveau afin d'obtenir un filtrat limpide.

5.4. Placer les tubes à essai dans l'eau en ébullition pendant $5 \mathrm{mn} \pm 2 \mathrm{~s}$.

\section{6) Interprétation des résultats}

6.1. Si le contenu du tube à essai reste limpide, on peut en conclure que le lait examiné est un lait stérilisé produit suivant les méthodes classiques. 
6.2. Si le contenu du tube à essai est trouble ou présente un précipité blanchâtre, on peut en conclure que le lait examiné est un lait U.H.T. ou un lait U.H.T. aromatisé ou chocolaté qui n'a pas été soumis à un pré-chauffage.

\section{Rés u m é}

Après avoir souligné qu'il n'existe actuellement aucune définition internationale du lait stérilisé, l'auteur pose les principes d'une définition correcte fondée sur la conservabilité, c'est-à-dire sur la stabilité physique et chimique, et non pas sur la stérilité qui est inutile, irréalisable d'une façon absolue et incontrôlable. ainsi :

Le projet de définition proposé par l'auteur peut se résumer

"Le lait stérilisé doit être exempt de germes pathogènes, et de » micro-organismes quelconques susceptibles de s'y développer. Il

" doit rester parfaitement stable pendant une durée commerciale" ment suffisante. Après trois semaines d'incubation à $31^{\circ} \mathrm{C} \pm 1^{\circ} \mathrm{C}$, " il ne doit pas renfermer, par ml, plus de 5 spores vivantes, inca» pables de proliférer dans le lait. Il doit être conditionné dans des » récipients étanches et entreposé à l'abri de la lumière ».

L'auteur, passant ensuite à l'étude des laits U.H.T., propose de les définir de la manière suivante :

« Laits stérilisés obtenus par chauffage en flux continu à haute " température (au moins $135^{\circ} \mathrm{C}$ ) pendant un temps très court (quel"ques secondes), conditionné aseptiquement en récipients stériles, " devant satisfaire au test de stabilité des laits stérilisés et au test "de turbidité d'Aschaffenburg modifié ».

Dans une deuxième partie, l'auteur pose les principes et décrit les méthodes de contrôle du lait stérilisé classique et du lait stérilisé U.H.T. permettant de vérifier leur conformité aux définitions précédentes.

\section{S u m m a ry}

After having underlined that, actually, there exist no international definition of sterilized milk, the author lays down the principles of a correct definition, based upon keeping qualities, that is upon physical and chemical stability and not upon sterility which is useless, unattainable in absolute sense and not verifiable.

The draft of definition proposed by the author may be summarized as follows :

"Sterilized milk should be free from pathogenic germs and " from any microorganism able to develop in it. It should remain " thoroughly stable for a commercially sufficient duration. After " three weeks of incubation at $31^{\circ} \mathrm{C} \pm 1^{\circ} \mathrm{C}$, it should not contain, 
" in one milliliter, more than 5 living spores unable to prolifer » in milk. It should be packaged in tight recipients and stored pro" tected from light ".

The author, studying the U.H.T. milks, propose to define them in the following manner :

« U.H.T. milks are sterilized milks obtained by an uninterrupted " continuous flow heating process involving a high temperature " treatment (at least $135^{\circ} \mathrm{C}$ ) for a very short period of time (a few » seconds), followed by aseptic packaging in sterile recipients. These " milks should pass the stability test for sterilized milks and the

" modified Aschaffenburg turbidity test ".

In a second part, the author lays down the principles and describes the control methods for classical sterilized milk and for sterilized U.H.T. milk, allowing to verify their conformity to the above definitions.

\section{Bibliographie}

[1] Bottazzi (V.) et al. (1966). - Scienza Tec. latt. casear, p. 133.

[2] Burton (H.) (1958). - Journ. Dairy Research, p. 75 et p. 324.

[3] Burton (H.). (1959). - C.R. du $15^{\mathrm{e}}$ Congrès International de Laiterie, vol. 4, p. 2045.

[4] Burton (H.) (1965). - Journal of the Society of Dairy Technology, vol. 18, $\mathrm{n}^{\circ} 2$.

[5] Buttiaux (R.) (1965). - La Stérilisation du Lait. Monographie F.A.O et O.M.S., n ${ }^{\circ} 65$, p. 205 à 216.

[6] Franklin (J. G.) et al. (1958). - J. Appl. Bact., 21, p. 47.

[7] Franklin (J.G.) et al. (1959). - C.R. du 15 Congrès International de Laiterie, vol. $1, \mathrm{p}, 410$.

[8] Galesloot (Th. E.), (1956). - Neth. Milk and Dairy Journal, 10, p. 79.

[9] Galesloot (Th. E.) et Radema (1957). - Rapp. Ned. Inst. Zuivelonderz., $\mathbf{n}^{\circ} 9$.

[10] GalesLoot (Th. E.) (1962). - Milk hygiene. Monographie F.A.O. et O.M.S., $\mathrm{n}^{\circ} 48$, p. 269.

[11] Hermier (J.) (1955). - Annales de Technologie, p. 341.

[12] Hermier (J.) (1957). - Annales de Technologie, p. 377.

[13] Hermier (J.) et al. (1959). - Le Lait, p. 20.

[14] KaPlan (A. M.) et al. (1954). - Food Research, p. 173.

[15] Lingren (B.) et SWARTLing (P.) (1963). - Milk and Dairy Research. Alnarp. Rapport $\mathrm{n}^{\circ} 69$.

[16] Moller-Madsen (A.) et al. - Beretn. St. Forsogsmeieri, no 151 et 152.

[17] PFeiffer (V.F.) et Vojnovich (C.) (1952). - Industr. Engineer. Chemistry, p. 1940 .

[18] PIEN (J.) (1957). - Le Lait, p. 169.

[19] Rambaud (M.) (1957). - Industr. Aliment. et Agricoles, p. 741.

[20] Tentoni (R.) (1955). - Il Latte, avril.

[21] Tentoni (R.) (1959), - C.R. du 15 Congrès International de Laiterie, vol. 1, p. 509.

[22] Thieulin (G.) et Vuillaume (R.). - Eléments Pratiques d'Analyse et d'Inspection du lait ( $3^{\circ}$ édition). Edition " Le Lait », 48, avenue du PrésidentWilson, Paris $16^{\mathrm{e}}$.

[23] THом́́ (K. E.) et al. (1964). - Milk and Dairy Research. Alnarp. Rapport $n^{\circ} 70$.

[24] Williams (D. J.) et al. (1957). - Journ. Appl. Bact., 20, p. 43. 